Grażyna Strzelecka

Universität Warschau, Institut für Germanistik https://orcid.org/0000-0003-2123-1273

g.strzelecka@uw.edu.pl

\title{
LOGISTIK AN DER GERMANISTIK - WARUM NICHT? EIN PLÄDOYER FÜR DEN FACHSPRACHENUNTERRICHT AN DER UNIVERSITÄT
}

\author{
Teaching logistics in German Studies - why not? \\ A request for specialized languages at university
}

Not often do we encounter a specialized foreign language course at a university humanities department. However, why should foreign language students not be provided with practical knowledge which may be useful in future professional life? The article presents an experiment conducted with the participation of MA students of German studies at Warsaw University. The aim is to teach language skills, as well as convey professional knowledge in the CLIL formula, which is becoming more and more popular. It is consistent with requests of students themselves. Logistics is a useful introduction to various branches of the economy. Considering that the German language is becoming more and more important in business-related professions, the topics discussed in this course may be required in logistics, forwarding and other areas. In addition to traditional logistics topics, such as transport, storage and waste disposal, we also discuss topics such as business correspondence or negotiations and practice translations.

Keywords: logistics, German studies, languages for specific purposes, university, CLIL

Słowa kluczowe: logistyka, filologia germańska, język specjalistyczny, uniwersytet, CLIL 


\section{Einleitung}

Fachsprachen im Fremdsprachenunterricht waren bis vor kurzer Zeit an geisteswissenschaftlichen Fakultäten eine Seltenheit. Doch auch an einer Philologie kann praktisches fachliches Wissen zusammen mit der Fremdsprache vermittelt werden: In dem Beitrag wird ein experimenteller Logistikunterricht in deutscher Sprache an der Warschauer Germanistik geschildert ${ }^{1}$. Die Logistik ist als Einstieg in diverse Bereiche des Wirtschaftslebens sehr gut geeignet: Es wird hier auf Themen aus dem Bereich Logistik und Spedition eingegangen sowie auf solche, die in vielen anderen beruflichen Bereichen, mit denen Absolventen der Germanistik in Berührung kommen, von Vorteil sein können. Neben typisch logistischer Thematik wie Transport, Lagerung und Entsorgung werden auch Themen wie Informationsmanagement, Management und Controlling sowie im schriftlichen Bereich die Geschäftskorrespondenz und Übersetzung behandelt. Diese Thematik kommt den Wünschen der Studierenden entgegen, da Deutsch als Fremdsprache in wirtschaftsorientierten Berufen immer mehr an Bedeutung gewinnt. So haben Inhalte, die im späteren beruflichen Leben praktisch eingesetzt werden können, für die Studierenden auch eine motivierende Funktion.

Eine von der Autorin in einer angehenden Logistikergruppe, die aus GermanistikstudentInnen im Aufbaustudium bestand, durchgeführte Umfrage ergab, dass das Wissen der Philologen über die Aufgaben der Logistik nicht umfangreich ist und die Definitionen, die sie jeweils angaben, sehr eingeschränkt. Die meisten behaupteten, die Logistik sei vor allem eine Transportaufgabe oder die Logistik bedeute schlicht den Transport von Gütern von einem zum anderen Ort. Die wenigsten hatten eine Vorstellung darüber, wie viele Aufgaben im Rahmen der Logistik bewältigt werden und wie oft man im Berufsleben mit ihr in Berührung kommt.

Nach bereits einem Semester Logistikunterricht, in dem Inhalt und Sprache (content and language) vermittelt wurden, waren die Antworten auf dieselben Fragen weit ausführlicher und zeugten nicht nur davon, dass die Studierenden sich Elemente einer Fachsprache angeeignet hatten, sondern auch, dass sie gewisse Fachinhalte gelernt und behalten haben. Diese lernten sie, wie sie angaben, „ganz nebenbei“ und sie hatten nicht den Eindruck, dass sie ihnen schwerfielen. Auf der anderen Seite lernten sie auch die Fremdsprache "irgendwie nebenbei“, da sie sich hauptsächlich auf die ihnen bis dato unbekannten Fachinhalte konzentrierten. Das Ergebnis war: Sprache und Inhalt, also CLIL (Content-and-Language-Integrated-Learning).

\footnotetext{
${ }^{1}$ Die erste Logistikgruppe wurde im Semester 2017/2018 unterrichtet. Zum Zeitpunkt der Fertigstellung des Beitrags konnten lediglich die Erfahrungen des Wintersemesters ausgewertet werden.
} 


\section{Was ist Logistik, was macht Logistik?}

Das Grundverständnis der Logistik als wissenschaftlicher Disziplin hat im Jahr 2010 der wissenschaftliche Beirat der Bundesvereinigung Logistik (BVL) in Form eines Positionspapiers erarbeitet. Logistik ist danach eine anwendungsorientierte Wissenschaftsdisziplin; es ist die ganzheitliche Planung, Steuerung, Koordination, Durchführung und Kontrolle aller unternehmensinternen und unternehmensübergreifenden Informations- und Güterflüsse. Synonym wird die Bezeichnung SCM (Supply Chain Management, also die intelligente Planung und Steuerung von Wertschöpfungsketten) verwendet.

Logistik analysiert und modelliert Wirtschaftssysteme als Flüsse von Objekten, Gütern und Personen in Netzwerken durch Zeit und Raum. Sie liefert Handlungsempfehlungen zu ihrer Gestaltung. Die Fragestellungen der Logistik beziehen sich auf die Konfiguration, Organisation, Steuerung oder Regelung dieser Netzwerke und Flüsse mit dem Anspruch, dadurch Fortschritte in der Erfüllung ökonomischer, ökologischer und sozialer Zielsetzungen zu ermöglichen. Logistik organisiert und steuert Lieferprozesse - vom Rohstoffabbau bis zur Ablieferung an den Endverbraucher.

Die vollständige Logistikdefinition der Bundesvereinigung Logistik (www.bvl.de, 26.10.2017) lautet:

Logistik ist ein System, das im Unternehmen, aber auch unternehmensübergreifend mit Lieferanten und Kunden, eine optimale Versorgung mit Materialien, Informationen, Teilen und Modulen für die Produktion - und auf der anderen Seite natürlich der Märkte bedeutet.

Eine der historischen Definitionen der Logistik, die besonders bekannt ist, ist die des amerikanischen Wirtschaftswissenschaftlers Edward Grosvenor Plowman: Die Logistik ist die Lieferung des richtigen Gutes, in der richtigen Menge, im richtigen Zustand, an den richtigen Ort, zur richtigen Zeit, für den richtigen Kunden und zu den richtigen Kosten. Diese Definition ist in die Wirtschaftsgeschichte als die „6 R der Logistik” eingegangen (www.bvl.de, 26.10.2017).

Lehrbücher geben zahlreiche Definitionen an, die mit den oben genannten übereinstimmen, wobei die Akzente unterschiedlich gesetzt werden. Fortmann und Kallweit (2007: 36) betonen die planenden, gestaltenden, abwickelnden und kontrollierenden Aufgaben:

Logistik ist die integrierte Planung, Gestaltung, Abwicklung und Kontrolle von Lagerungs- und Transportprozessen innerhalb und zwischen Unternehmen, oder vom Unternehmen zum Kunden, sowie der Entsorgungswege. Das Ziel der Logistik ist diese einzelnen Aufgaben optimal zu verknüpfen. 
In der Logistik geht es um Steuerung eines materiellen und immateriellen Warenstroms innerhalb eines Unternehmens sowie zwischen Unternehmen, Lieferanten und Endkunden. Während zu materiellen Dingen Rohstoffe, Zwischenprodukte, Endprodukte sowie Strom, Wasser, Schmiermittel, auch Maschinen, Transport- oder Lagermittel zählen, so werden zu immateriellen Dingen, die zur Bewältigung der Logistik-Aufgaben notwendig sind, prozessorientiertes Denken, Kommunikation und Information oder die Informationsübertragung gerechnet. Spätestens an diesem Punkt ergibt sich eine Schnittstelle zu den Sprachen, insbesondere zu den Fremdsprachen und ihrem Einsatz für die Kommunikation in der Wirtschaft. Um logistische Aufgaben zu bewältigen, ist beides notwendig: Fachkenntnis und Sprachkenntnis. Diese Bereiche sind eng miteinander verflochten. Erst wenn beides vorhanden ist, kommt eine gute fachliche Kommunikation zustande. Eine solche Kommunikation können einerseits Fachleute, andererseits aber auch Philologen verwirklichen.

\section{Warum Logistik?}

\subsection{Karrierestart in der Logistikbranche}

Die Logistik zeigt einen Querschnitt durch die Aktivitäten eines Unternehmens und seine Beziehungen zur Außenwelt: Der Einstieg in die Logistik ist für Studierende an geisteswissenschaftlichen Fakultäten zunächst nicht einfach, doch er bietet sowohl eine sehr gute sachliche als auch eine sprachliche Vorbereitung für berufliche Tätigkeiten in unterschiedlichen Positionen. Logistik zeigt auch Berufsfelder, die der eine oder andere Lerner tatsächlich später wählen kann, denn gerade Philologen sind es, die nach ihrem Studium sehr unterschiedliche Berufe ergreifen und in unerwarteten Positionen arbeiten.

Das Arbeitsfeld für Logistiker ist sehr breitgefächert. Nach den LogistikTipps der Absolventa (www.absolventa.de, 10.11.2017) legt man als Student den Grundstein für die Logistik-Karriere am besten mit einem Logistik-Praktikum. So erhält man frühzeitig Einblicke beispielsweise in Lagerlogistik-Aufgaben oder man lernt, was es bei Transport-Jobs zu beachten gilt. Wer nach dem wirtschaftswissenschaftlichen Studium auf den Arbeitsmarkt kommt, kann in den Logistik-Stellenangeboten nach attraktiven Jobs suchen. Auch viele Absolventen der philologischen Fakultäten werden an Unternehmen beschäftigt, in denen logistische Prozesse tagtäglich vor sich gehen. Sie zu verstehen und in der Fremdsprache richtig benennen zu können, wird ihre Chancen auf dem Arbeitsmarkt steigern, denn sie werden jedem Arbeitgeber willkommen sein. Deshalb ist Logistik ein sehr geeigneter Wirtschaftsbereich, um Studierende auf ihren Einstieg in den Arbeitsmarkt vorzubereiten. Logistische Prozesse bereits im 
Studium verstehen zu lernen, wird das Verstehen der Arbeitswelt - so wie sie nach dem Abschluss vor zu finden sein wird - fördern. Wer später möglicherweise andere Wirtschaftsbereiche zu seinem Berufsfeld zählt, wird nach einem Logistikkurs für viele davon bestens vorbereitet sein.

\subsection{Wünsche der Arbeitgeber und der Arbeitnehmer}

Mehrere Wissenschaftler erforschten die Wünsche der Arbeitgeber hinsichtlich der Fremdsprachenkenntnisse und kommunikativen Fähigkeiten ihrer zukünftigen Arbeitnehmer (Held, 2015: 71-80). Dabei erwähnten die Arbeitgeber vor allem drei Bereiche, in denen die idealen Arbeitnehmer in der Fremdsprache bewandert sein sollten:

1. Kommunikation,

2. Geschäftskorrespondenz,

3. Verhandlungen.

Die Studierenden ihrerseits erwähnten folgende Bereiche, die sie in der Fremdprache gerne vermittelt bekommen würden:

1. Sachwissen,

2. Fachkenntnisse,

3. Kommunikation.

Anhand der Untersuchungen kann man feststellen, dass die Wünsche der Studierenden mit den Wünschen der Arbeitgeber weitgehend übereinstimmen; die Ergebnisse weisen auch auf die erwünschte Richtung des Fremdsprachenunterrichts in der Zukunft hin. Die Logistik als Fach wird den Wünschen der Arbeitgeber und der zukünftigen Arbeitnehmer bezüglich der Fremdsprachenkenntnisse mehr als gerecht.

\subsection{Wirtschaftsdeutsch}

Eine Frage, die oft aufkommt, wenn man zur praktischen Verwirklichung der Wunschlisten der Arbeitgeber und Arbeitnehmer übergeht, ist: Können wir Fachsprachen an der Germanistik unterrichten? Welche Fachsprachen sollen es sein? Wir wissen nicht, welche Berufe unsere Absolventen letztendlich ergreifen. Viele gehen in die Wirtschaft oder in den öffentlichen Sektor und bekleiden dort unterschiedliche Ämter. Andere werden Lehrer, Übersetzer und Dolmetscher. Die grundsätzliche Frage ist also: Was können wir ihnen auf ihren beruflichen Weg mitgeben? Abhängig von der bekleideten Position braucht jeder einen anderen Fremdsprachenunterricht, denn er wird andere Fähigkeiten und Kenntnisse in seinem Berufsleben einsetzen müssen (Buhlmann, Fearns: 2000: 305) Welche Grundlagen für ihre unterschiedlichen zukünftigen 
Berufe können wir den Studierenden im Fremdsprachenunterricht bieten? Hinsichtlich des Fremdsprachenunterrichts an Universitäten ist der Begriff Wirtschaftsdeutsch ein Schlüsselwort. Nach Buhlmann und Fearns (2000: 306) ist es ein Sammelbegriff für mehrere Fachsprachen und Bereiche:

Wirtschaftsdeutsch ist ein Sammelbegriff für diverse Fachsprachen, die von Personengruppen mit unterschiedlicher Vorbildung, unterschiedlichen Tätigkeiten und unterschiedlichen Kommunikationszielen und -formen in einem beruflichen, akademischen und/oder ausbildungsbedingten Umfeld benutzt werden, das irgendwie mit Wirtschaft zu tun hat.

Im Rahmen des Faches Wirtschaftsdeutsch gibt es unterschiedliche Sprachverwendungsbereiche, zwischen denen nicht unbedingt Gemeinsamkeiten bestehen. In Bezug auf Personen, die im Wirtschaftsleben agieren, wird festgestellt, dass sie je nach dem von ihnen ausgeübten Beruf, über unterschiedliche Kenntnisse und Fähigkeiten verfügen müssen. Auch diesbezüglich bietet das Fach Logistik ein breites Spektrum an Möglichkeiten.

Mehrere Forscher und Fremdsprachendidaktiker in Polen beschäftigen sich seit Jahren mit dem Begriff "Wirtschaftsdeutsch“ und widmen zahlreiche Veröffentlichungen der Fachsprachendidaktik. Diesbezüglich gibt es mehrere Veröffentlichungen von der Romanistin Magdalena Sowa, die insbesondere die Anpassung der Lehrprogramme an den Hochschulen an die aktuellen Erfordernisse des Arbeitsmarktes betont (Sowa:2015: 117). Ein wichtiges Werk, das in den letzten Jahren erschienen ist, ist die Monographie von Elżbieta Gajewska und Magdalena Sowa unter dem Titel „LSP, FOS, Fachsprache. Dydaktyka Języków Specjalistycznych" (Gajewska, Sowa: 2014), ein Kompendium, das die Gedanken und Bestrebungen zum Fachsprachenunterricht insbesondere an Fachhochschulen und Universitäten zusammenfasst. Es werden dort auch die Methoden und Instrumente zum Fachsprachenunterricht erfasst und beschrieben. Betont wird dabei der aufgabenorientierte Fremdsprachenunterricht. (Sowa, Gajewska: 2014: 168), der die Eigenkreativität der Lernenden fördert und somit ihre Autonomie und Aktivität steigert. Dies knüpft an die bereits in den 80-iger Jahren des 20. Jahrhunderts bekannte Methode TBLT (Task Based Language Teaching) an.

In Übereinstimmung damit betonen mehrere Fachsprachenlehrer an wirtschaftsorientierten Universitäten in Polen den sich langsam vollziehenden Übergang vom allgemeinsprachlichen Unterricht zum Fachsprachenunterricht (Zielińska, 2015: 53). Dies ist überdies in den größeren Kontext der Diskussion um die allgemeine Gestalt des Hochschulwesens und der Frage nach dem praxisorientierten Hochschulwesen eingebettet (Parczewski, 2015: 81). Anlässlich 
des Jubiläumsjahres der Warschauer Universität fand eine eigens zu dieser Frage organisierte Konferenz statt².

Einige Hochschullehrer führen detaillierte Umfragen unter den Studierenden an ihren Hochschulen durch, um in Erfahrung zu bringen, was ihre Wünsche und Bedürfnisse bezüglich des Fremdsprachenunterrichts an ihrer Hochschule sind. Für die englische Sprache führten dies beispielsweise die Autorinnen Anna Żebrowska und Jolanta Idźkowska durch. Aufgrund der durchgeführten Analysen kamen sie zu dem Schluss, dass Fachsprachenkurse gerade im Aufbaustudium sehr motivierend wirken (Żebrowska, Idźkowska: 2016:187). Sie wiesen dabei darauf hin, dass die große Anzahl von Fachbereichen eine Quelle der Schwierigkeiten für den Fachsprachenunterricht und die Fachsprachenlehrer darstelle (Żebrowska, Idźkowska: 2016:188). In der Umfrage bestätigten dies über $90 \%$ der Lehrbeauftragen. Dies knüpft an die These des Fachsprachenforschers Hans Rüdiger Fluck an, dass heutzutage die Zahl der Spezialisierungen nicht mehr zu überblicken ist. Universale Kenntnisse zu erwerben ist nicht mehr möglich, und der Einzelne hat über die einzelnen Wissensbereiche keinen Überblick mehr. Der Fachwortschatz zahlreicher Disziplinen nimmt schnell zu und zeigt dabei wie die Gebiete selbst eine zunehmende Spezialisierung. Schon im 20. Jahrhundert musste so viel Neues benannt werden wie noch nie zuvor (Fluck, 1998:31). Diese Beschreibung trifft auf die Sprache der Wirtschaft zu und auch das ist ein Argument für den Logistikunterricht, da die Logistik wie keine andere Fachdisziplin einen Querschnitt durch mehrere Fachbereiche bietet.

\subsection{Wirtschaftsdeutsch an der Warschauer Germanistik}

Das Fach „Wirtschaftsdeutsch“ wurde an der Warschauer Germanistik unter dem Namen "Spezialthema“ bereits in den 90-er Jahren eingeführt, zum Zeitpunkt der politischen und wirtschaftlichen Umwälzungen. Mehrere Unternehmen aus Deutschland und Österreich stürzten auf den polnischen Markt und eröffneten nacheinander ihre Büros in der Hauptstadt: Bosch (1991), Siemens (1991), Bayer (1991), Henkel (1991) MAN (1993), Metro (1994), Mercedes (1996), danach Media Markt, Saturn und viele weitere. Man suchte Mitarbeiter mit guten Deutschkenntnissen und stellte Germanistikabsolventen an, deren „literarisches" Deutsch aber nicht immer zu der Sprache passte, die man in den Unternehmen sprach. So wurde nach und nach der Themenbereich des Wirtschaftsdeutschunterrichts an der Germanistik um immer neue

\footnotetext{
${ }^{2}$ Humanistyka a praktyczny profil kształcenia uniwersyteckiego w nauczaniu języka, literatury i kultury w warunkach obcokulturowych, Uniwersytet Warszawski , 2015
} 
Themen erweitert: Es waren: Export, Import, Werbung, Handel, Marketing, Banken, Börse, Finanzen sowie Geschäftskorrespondenz und Verhandlungen. Heute wird an der Germanistik Wirtschaftsdeutschunterricht mit mehreren Schwerpunkten in der CLIL-Formel (integriertes Lernen von Fremdsprachen und Fachinhalten) realisiert. Die Anknüpfung an CLIL bedeutet, dass es im praktischen Deutschunterricht nicht allein um Sprache, sondern auch um Inhalte aus verschiedenen Bereichen geht, die hier vermittelt werden. Aktuelle Themenbereiche an der Warschauer Germanistik sind: Werbung, Tourismus, Marketing, Business und Management, Logistik sowie Spezialthemen von außerhalb des Wirtschaftsbereiches, wie Pressesprache und Amtsdeutsch.

\subsection{Wirtschaftshochschule versus Universität}

Für den Wirtschaftsdeutschunterricht ist der Unterschied zwischen den Studierenden an einer Wirtschaftshochschule und den Studierenfen an einer Universität, bzw. den Studierenden an einer Wirtschaftsfakultät und den an einer geisteswissenschaftlichen Studienrichtung gravierend: Die einen besitzen das erforderliche wirtschaftliche Basisfachwissen und die anderen nicht. Der/die Studierende an einer Fakultät an der Wirtschaftshochschule SGH (Szkoła Główna Handlowa) in Warschau wird über grundsätzliches Wirtschaftsfachwissen verfügen; dieses muss ihm/ihr im Fremdsprachenunterricht nicht mehr vermittelt werden. Der/die Studierende an der philologischen Fakultät der Warschauer Universität wird höchstwahrscheinlich darüber nicht verfügen. Der Dozent/die Dozentin wird es auch nicht verlangen können, sondern er/sie wird es im Unterricht selbst vermitteln müssen. Dies ist ein völlig anderer Ausgangspunkt, der den Lernprozess und die Motivation zum Erlernen einer Fremdsprache mit fachsprachlichen Aspekten beeinflusst (Strzelecka, 2018). Gerade die Motivation, neben der Fremdsprache auch fachliche Inhalte vermittelt zu bekommen, spielt im Fremdsprachenunterricht für Philologen eine nicht zu unterschätzende Rolle.

\subsection{Motivation}

Das Erlernen der Fachsprache anhand realer Situationen aus dem Berufsalltag gestaltet den Lernprozess interessanter, attraktiver und „benutzerfreundlicher" als im allgemeinsprachigen Unterricht. Dies war das Ergebnis der erwähnten Umfrage nach einem Semester Logistikunterricht. Das Berufsleben bietet viele Situationen, die im Unterricht in Form von Dialogen, spontanen Rollenspielen oder Fallstudien (case studies) eingesetzt werden können. Bei Studierenden an geisteswissenschaftlich orientierten Fakultäten kann nicht 
davon ausgegangen werden, dass sie Kenntnisse im Bereich Logistik verfügen. Sie müssen hier und jetzt die logistischen Schlüsselbegriffe auf Deutsch lernen und sie später anwenden können. Sie üben es unter anderem, indem sie die Schlüsselbegriffe aus einem Text erklären und sie übersetzen, logistische Aufgaben in Form von Fallstudien in der Fremdsprache lösen oder verschiedene Rollen in Wirtschaftsgesprächen übernehmen. Die für den Unterricht gewählten Texte und Dialoge stammen aus originellen Materialien zur Logistik bzw. aus der deutschsprachigen Presse oder sind dem authentischen Wirtschaftsleben entnommen/nachgestellt ${ }^{3}$. Das alles fördert die Motivation zum Lernen und den Willen, sich „nebenbei“ auch Wirtschaftswissen anzueignen.

\subsection{Ziele der Berufsbildung}

An dieser Stelle sollte kurz auf das Ziel der Berufsbildung eingegangen werden, die Lerner auf die sich verändernde Wirtschafts- und Berufswelt vorzubereiten, damit sie ihren Beruf so gut wie möglich ausüben und auf dem sich immer weiter entwickelnden Arbeitsmarkt lange aktiv bleiben. Die spezifischen Berufsziele der Branche Logistik an einer Schule bzw. Hochschule mit Spezialfach Logistik sind (Suszczyńska, 2018):

- Organisationarbeit und Verladung der Waren planen,

- Dokumentation der Transportprozesse führen,

- Dokumentation über Verrechnungen mit Kunden und ausländischen Partnern führen,

- Transportprozesse überwachen,

- Lagerungsprozesse organisieren und überwachen,

- Distribution der Waren planen und kontrollieren,

- technische Mittel im Transport verwalten und sie für die Abwicklung der Transportprozesse organisieren.

Die Kenntnis dieser Ziele gibt dem Fremdsprachenlehrer wichtige Hinweise dafür, was er im Fremdsprachenunterricht einführen kann und auf welche Aspekte er sich konzentrieren soll. Im Fremdsprachenunterricht erwirbt der Lernende nämlich weitere Qualifikationen, so dass er nach Kursabschluss Folgendes kann: die Sprachmittel (lexikalische, grammatische, orthografische und phonetische) für die Abwicklung der beruflichen Aufgaben anwenden, typische Aussagen über Berufstätigkeiten formulieren und interpretieren, kurze Texte über typische Berufstätigkeiten analysieren sowie kurze, verständliche

${ }^{3}$ Für den an der Germanistik neu eingeführten Logistikunterricht gibt es kein Lehrbuch. Der Lehrbeauftragte stellt die Materialien zusammen, dabei können auch Wünsche der Studierenden berücksichtigt werden. 
berufsbezogene Texte selbst formulieren, darüber hinaus fremdsprachige Informationsquellen auswerten (Suszczyńska, 2018).

Auch der Sprachunterricht im Studium, so auch an der Germanistik, sollte diesen Zielen gerecht werden. Der Absolvent der Germanistik, der meistens vor Aufnahme des Hochschulstudiums ein Allgemeinbildendes Lyzeum besuchte (Absolventen von technischen Oberschulen sind an philologischen Fakultäten äußerst selten), kann hier in der CLIL-Formel das notwendige Fachwissen erwerben und gleichzeitig seine Fremdsprachenkenntnisse erweitern und vervollkommnen.

\section{Logistik an der Warschauer Germanistik}

\subsection{Ziel und Inhalt}

Das Ziel der Lehrveranstaltung Logistik an der Warschauer Germanistik ist die Studierenden mit der Fachsprache vertraut zu machen, die in Dienstleistungssektoren wie Logistik und Spedition verwendet wird sowie das Kennenlernen des Fachwortschatzes und der Wendungen, die eine erfolgreiche Kommunikation in Wort und Schrift ermöglichen. In der Lehrveranstaltung lernen die Studierenden Wendungen kennen, die notwendig sind, um erfolgreich in verschiedenen Bereichen der Logistik und Spedition zu kommunizieren. Dies ermöglicht ihnen Texte und Briefe zu verfassen, den Weg eines Produktes vom Produzenten zum Konsumenten zu verfolgen und zu beschreiben, Statistiken, Berichte und Diagramme zu lesen und zu interpretieren sowie Dokumente auszufüllen und selbst herzustellen. Das Programm umfasst Elemente des Marketings und des Engagements der Logistik in die Verantwortung für die Umwelt (Grüne Logistik), einen praktisch angelegten Kurs der Geschäftskorrespondenz und diverse Übersetzungsübungen, die der Aneignung des Fachwortschatzes dienen.

Die Studierenden lernen aktuelle Themen aus der Presse kennen, üben die Durchführung von Telefongesprächen und die Zusammenstellung von logistischen Wegen in Form von case studies. Bestandteil der Lehrveranstaltung sind ausgewählte Fragen aus dem Bereich der Kontaktpflege mit Vertretern von Ämtern (Zollamt, Polizei, Konsulat).

\subsection{Bildungseffekte}

Die erwarteten Bildungseffekte, die mit den Europäischen Rahmenbedingungen übereinstimmen, sind:

- die gehörte oder gelesene Aussage samt Fachwortschatz (aus der logistischen Branche) aus dem betreffenden Bereich zu verstehen, 
- eine Analyse des Textes, die Interpretation eines Diagramms oder einer Aussage vorzunehmen und die gewonnenen Informationen für die Erstellung eigener Texte zu nutzen sowie Informationen aus verschiedenen Quellen zu selektieren,

- statistische Angaben zur Logistik zu interpretieren und die gehörten oder gelesenen Aussagen aus verschiedenen Quellen zu vergleichen,

- in einem Telefongespräch spontan zu reagieren sowie schriftlich Inhalte auszudrücken, die der Promotion von Produkten und (logistischen) Dienstleistungen dienen,

- die Kenntnis des Fachwortschatzes und diverser Bedeutungsnuancen zu zeigen. Wörter und Wendungen zu kennen, die in der Logistik anwendbar sind,

- beim Verfassen von Texten entsprechende stilistische und formelle Mittel anzuwenden und einen Text mit Berücksichtigung entsprechender Stilebenen übersetzen zu können,

- in Wort und Schrift eine Aussage zu verfassen, die auf das Erreichen eines ganz bestimmten, angestrebten (logistischen) Ziels zielt.

Diese Effekte wurden aus Sicht der Autorin bereits in dem ersten an der Germanistik durchgeführten Kurs zur Logistik größtenteils erreicht. Insbesondere im Bereich der Eigenproduktion von Texten und Aussagen mit logistischen Inhalten konnten weitgehende Erfolge verzeichnet werden. Die Studierenden hatten auch sichtlich Spaß am Einsatz von Fachwortschatz, was andernorts meistens als "schwierig“ bezeichnet wird. Da zum Zeitpunkt, zu dem dieser Beitrag erscheint, noch keine langfristigen Resultate messbar sind, bleibt zu hoffen, dass sie auch langfristig erreicht werden und die erworbenen Kenntnisse von den Studierenden im späterren Berufsleben eingesetzt werden können, was den Sinn dieser Veranstaltung ergibt.

\section{Zusammenfassung und Ausblick}

Die Logistik ist ein weiter, fachübergreifender Bereich, der für den Fremdsprachenunterricht an einer geisteswissenschaftlichen Fakultät sehr gut geeignet ist. Am Beispiel der Logistik wurde hier gezeigt, dass man an der Philologie erfolgreich sachbezogenen Fremdsprachenunterricht in der Content-and-Language-Formel einsetzen kann. Aufgabe des Dozenten ist, neben der Fremdsprache auch Wirtschaftswissen, über das seine Studentinnen nicht verfügen, zu vermitteln. Die Einführung von Fachsprachen an geisteswissenschaftlichen Fakultäten (hier am Beispiel der Logistik) fördert den Fremdprachenunterricht, denn:

- Sachinhalte, die im Unterricht vermittelt werden können, bieten Anreize dazu, das erworbene Wissen selbständig zu vertiefen und zu erweitern; Kritisches und kreatives Denken wird gefördert, 
- Logistik kommt den Erwartungen der Arbeitgeber entgegen, die von einem neuen Mitarbeiter in einem Großunternehmen erwarten, dass er die grundlegenden Abteilungen des Betriebs und ihre Aufgaben sowie die Zusammenhänge des Unternehmens mit der Außenwelt erkennt,

- Logistikspezifische Themen sind für die Studierenden neue Felder, die auf die Spezifik diverser Jobs in großen und kleinen Unternehmen vorbereiten,

- Die über die Logistik hinausgehenden Themen sind eine allgemeine praktische Vorbereitung für die Übernahme diverser schriftlicher und mündlicher Aufgaben durch den Absolventen, der einen der Berufe in der Wirtschaft ergreift,

- Der Erwerb sachbezogener Inhalte ist eine motivierende Herausforderung,

- Die kommunikativen Fähigkeiten in einem bestimmten Fachbereich (hier: Logistik) eröffnen neue berufliche Perspektiven,

- Die praktischen Kenntnisse im Umgang mit den Unternehmen und Geschäftspartnern sind von großem Wert im späteren Berufsleben, auch wenn der Absolvent einen anderen Dienstleistungsbereich oder Wirtschaftszweig wählt.

Daher sollten Fachsprachen aus weiten, durchgreifenden Bereichen wie die Logistik in Fremdsprachenprogramme einziehen und Bestrebungen, im Fremdsprachenunterricht Fachinhalte aus verschiedenen Wirtschaftsbereichen zu vermitteln und die Fremdsprache berufsorientiert zu unterrichten, sollten seitens der Hochschulbehörden Unterstützung finden. Dies soll ein Hinweis auch darauf sein, dass Schulungen für CLIL-Lehrkräfte wünschenswert wären, damit sie Kompetenzen erwerben und weiterentwickeln können, um in der CLIL-Formel erfolgreich zu unterrichten. Im Kontext des Qualifikationsrahmenprogramms der EU wird Kompetenz als Verantwortung einerseits und und Autonomie andererseits beschrieben (Europäische Komission, 2008). Das heisst, dass der Dozent/die Dozentin auch eigene Ideen einsetzen und eigene Unterrichtspraktiken verwirklichen kann, doch neben diesem Selbstmanagement braucht er/sie auch institutionelle Unterstützung.

Für die Forschung ergeben sich aus der Thematik mehrere Forschungsbereiche, die den Einsatz von Fachsprachen im Fremdsprachenunterricht betreffen, u.a. die Rolle der Motivation bei Studierenden an geisteswisseschaftlichen Fakultäten, die in der CLIL-Formel neben der Fremdsprache Fachinhalte erlernen.

Für den zukünftigen Fremdsprachenunterricht an geisteswissenschaftlichen Fakultäten, darunter an Philologien, wäre es wünschenswert, breitgefächert fachsprachlichen Unterricht einzuführen. Entgegen dem verbreiteten Vorurteil, dass die fachlich orientierten Inhalte im Gegensatz zum allgemeinsprachlichen Unterricht stünden, ergänzen sie ihn und erweitern um neue, 
motivierende Aspekte. Für die Universität ist die Ausarbeitung eines Bildungssystems, das neben dem Wissenstransfer und der intellektuellen Entwicklung auch die Entwicklung der Fähigkeit berücksichtigt, das erworbene Wissen nach dem Studium praktisch im Beruf umzusetzen (auch und eben in der Fremdsprache), eine zukünftige Herausforderung. Der Sprachunterricht an den philologischen Fakultäten kann und muss neu orientiert werden: Der Beitrag ist eine Stimme für den Fachsprachenunterricht an der Universität.

\section{LITERATURVERZEICHNIS}

Buhlmann R., Fearns A. (2000), Handbuch des Fachsprachenunterrichts: Unter besonderer Berücksichtigung technischer Fachsprachen. Tübingen: Narr Studienbücher.

Fortmann K.M., Kallweit, A. (2000), Logistik. Klausur Intensiv Training BWL. Stuttgart: Kohlhammer Verlag.

Held M. (2015), Czego pragnie pracodawca? Czyli rzecz o wymaganiach wobec pracowników i kandydatów w dziedzinie języków obcych oraz umiejętności komunikacyjnych (in) Srebro M., Typek E., Zielińska L. (Hrsg.), Przyszłość nauczania języków obcych na uczelniach wyższych. Kraków: Uniwersytet Ekonomiczny, S. 71-80.

Fluck H-R. (1998), Fachsprachen und Fachkommunikation. Heidelberg: Gross.

Gajewska E., Sowa M. (2014), LSP, FOS, Fachsprache..., Dydaktyka języków specjalistycznych. Lublin: Werset.

Parczewski P. (2015), Dekonstrukcja Krajowych Ram Kwalifikacji - krytyczna analiza wpływu Krajowych Ram Kwalifikacji na poprawę jakości nauczanai języków obcych w polskich szkołach wyższych (in) Srebro M., Typek E., Zielińska L. (Hrsg.). Przyszłość nauczania języków obcych na uczelniach wyższych. Kraków: Uniwersytet Ekonomiczny, S. 81-96.

Sowa M. (2015), Ewolucja programu kształcenia na filologii romańskiej w swietle wyzwań rynku pracy i Krajowych Ram Kwalifikacji (in) Srebro M., Typek E., Zielińska L. (Hrsg.). Przyszłość nauczania języków obcych na uczelniach wyższych. Kraków: Uniwersytet Ekonomiczny, S.111-122.

Strzelecka G. (2015), Wirtschaftsdeutsch für Germanistikstudenten: eine Gratwanderung zwischen Praxis und Ideal (in) Srebro M., Typek E., Zielińska L. (Hrsg.), Przyszłość nauczania języków obcych na uczelniach wyższych. Kraków: Uniwersytet Ekonomiczny, S.123-124.

Strzelecka G. (2017), Wirtschaftsdeutsch für Germanistikstudenten: Peripherie oder ein Zeichen der Zeit? Einige Erwägungen zu den Bereichen Touristik und Logistik (in) Janikova V., Nalepova J. (Hrsg.), Zentrum und Peripherie. Aus Fremdsprachendidaktischer Sicht. Opava: Slezska univerzita v Opavê, S.141-156. 
Strzelecka G. (2018), Wozu brauchen wir die Logistik? Einige Überlegungen zum Fachsprachenunterricht (in) Krawiec M., Pitchard R. (Hrsg.), Seize the Day: new perspectives on Foreign Language Learning and teaching. Hamburg: Verlag Dr. Kovač (im Druck).

Strzelecka G., Suszczyńska R. (2016), Język niemiecki zawodowy w logistyce i spedycji, Warszawa: Wydawnictwa Szkolne i Pedagogiczne.

Suszczyńska R. (2018), Das Lehrbuch „Fachsprache Deutsch in Logistik und Spedition" - eine kommentierte Besprechung (in) Krawiec M., Pitchard P. (Hrsg.) Seize the Day: new perspectives on Foreign Language Learning and teaching, Hamburg: Verlag Dr. Kovač (im Druck).

Zielińska L. (2015), Content and Language Integrated Learning approach in Teaching Foreign Languages at the University Level (in) Srebro M., Typek E., Zielińska L. (Hrsg.) Przyszłość nauczania języków obcych na uczelniach wyższych. Uniwersytet Ekonomiczny, S. 53-68.

Internetquellen

www.bvl.de (letzter Zugriff: 2.01.2017)

www.absolventa.de (letzter Zugriff: 4.01.2017). 